\title{
Study of the Drell-Yan process with the pair-production of polarized tau leptons in collisions of polarized proton beams
}

\author{
A. Aleshko ${ }^{1, *}, E$. Boos $^{2, * *}$, and $V$. Bunichev ${ }^{2, * * *}$ \\ ${ }^{1}$ Lomonosov Moscow State University, 119991 Moscow, Russia \\ ${ }^{2}$ Skobeltsyn Institute of Nuclear Physics, MSU, 119991 Moscow, Russia
}

\begin{abstract}
The new method for studying polarized parton distribution functions via the Drell-Yan process with tau production is proposed. The hadronic decay of tau to single charged pi-meson and neutrino is utilised for determination of the polarization state of the tau-lepton. The key feature of our approach is that we do not sum over the polarizations states of tau, but preserve this information through energies of pions produced in corresponding decays. The new method in addition to existing ones should improve the accuracy of measurements of polarized structure functions of hadrons. In the current work, the feasibility of such an approach is assessed through numerical simulations.
\end{abstract}

\section{Introduction}

During the past decades, the parton-quark model of the proton was developed. According to this model, the proton consists of three valence quarks and the cloud of virtual gluons and quark-antiquark pairs. In collisions at high energies quarks are "asymptotically free", which means that the interaction between two quarks can approximately be treated as between two free particles. The probability of the process with a particular parton is described by the function, which is dependent on the fraction of the total momentum of the proton carried by that parton and the momentum transfer scale. These functions are known as parton distribution functions (PDF) and contain information about the internal structure of corresponding nucleons $[1,2]$. If one is to account for possible transverse momenta of quarks, additional transverse momentum dependent (TMD) PDFs are also to be introduced.

Hence, for a consistent description of processes with polarized nucleons in a wide energy range and the most complete description of the proton structure a generalized parton model was developed [3-5], which consist of 8 specialized parton distribution functions. These functions are: the distribution of the parton density in unpolarized nucleon (Density); distribution of the longitudinal polarization of quarks in the longitudinally polarized nucleon (Helicity); distribution of transverse polarization of quarks in transversely polarized nucleon (Transversity); the correlation between the transverse polarization of a nucleon and the transverse momentum of unpolarized quarks (Sivers); the correlation between the transverse polarization of a nucleon and the longitudinal polarization of quarks (Worm-gear-T);

\footnotetext{
*e-mail: isserq@gmail.com

**e-mail: boos@theory.sinp.msu.ru

***e-mail: bunichev@theory.sinp.msu.ru
} 


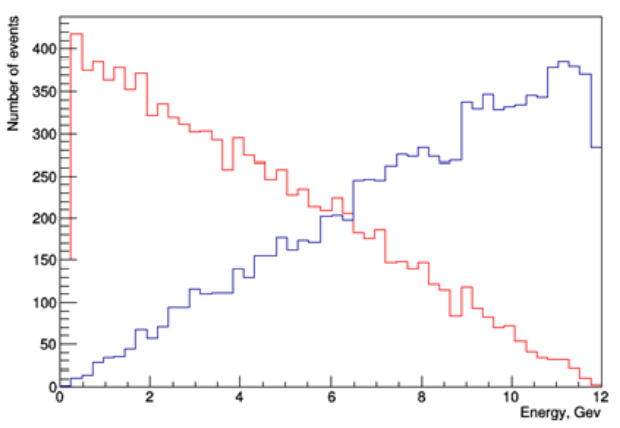

Figure 1. The energy spectra of the pi-meson produced from decays of taus which are: mostly lefthanded (red), mostly right-handed (blue)

the distribution of the transverse momenta of quarks in unpolarized nucleon (Boer-Mulders); the correlation between longitudinal polarization of nucleon and the transverse momenta of quarks (Worm-gear-L); the distribution of the transverse momenta of quarks in transversely polarized nucleon (Pretzelosity).

At the current level of our knowledge of the nucleon structure, parton distribution functions cannot be obtained analytically and have to be measured experimentally. The deep inelastic scattering (DIS,SIDIS) and the Drell-Yan (DY) processes are typically used for the measurements of PDFs [6-11, 11-18]. In the next section, we will consider the Drell-Yan process as a tool for the extraction of PDFs.

\section{Drell-Yan process with tau-production}

In the Drell-Yan processes, the annihilation of a quark and antiquark, from colliding nucleons, through an intermediate photon or Z-boson leads to the production of a lepton-antilepton pair $[11,19]$. The matrix element of the Drell - Yang process consists of a convolution of two tensors $[11,20,21]$. The first one is responsible for the hadron part with initial partons and includes a dependence on the polarized parton distribution functions. The second tensor corresponds to the lepton part of the process. Such a simple structure and the fact that DrellYan process is one of the cleanest hard hadron-hadron scattering processes makes it a very convenient tool for studying PDFs.

The conventional way for studying parton distribution functions via Drell-Yan process is to use processes with the production of electron-positron (and muon anti-muon) pairs [11, $16,18]$, because in this case the mass of leptons can be neglected and one can consider them as having some definite helicity. Within this approach angular parameters, which define the spatial orientation of the momenta of leptons relative to the momenta of colliding nucleons, are used as parameters describing the polarization state of the lepton pair. Then one can measure azimuthal asymmetries and extract particular structure functions from them (which is a considerable theoretical challenge).

In the case of tau production, this approach will work only at very high energies. At lower energies, however, the mass of tau cannot be neglected. Massive particles do not have definite helicity. Therefore, for tau leptons, the angular parameters as spin-sensitive variables can be used only at collision energy much greater than the mass of the tau lepton and are not suitable for energies comparable to its mass. Consequently, we need a more suitable parameterization of the polarization state of tau lepton. 

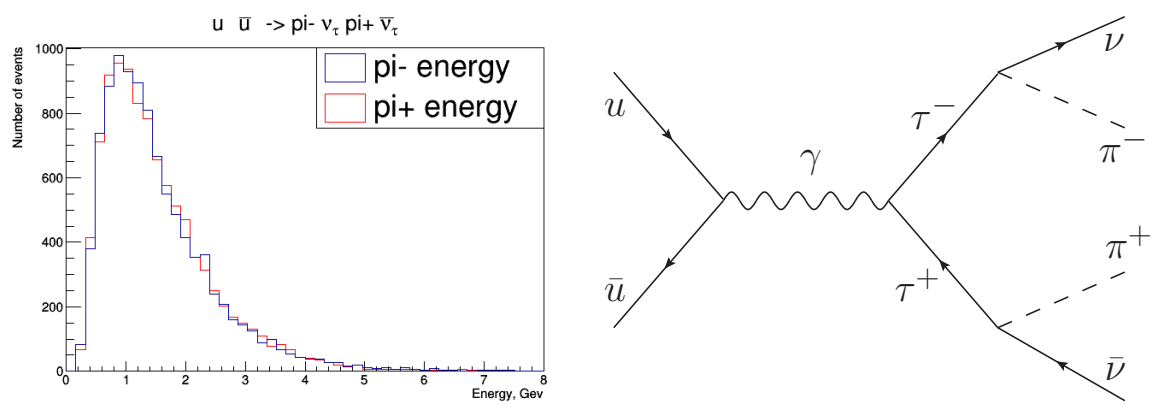

Figure 2. Energy spectra of pi-mesons in unpolarized case (left); the diagram for the simulated process (right).

Our idea to tackle this problem is to utilize the unique decays properties of tau-lepton. Consider the hadronic decay of tau to a single charged pi-meson and neutrino. Due to the weak nature of this decay and the fact that neutrinos are always left-handed, the energy spectra of pi-meson is strictly correlated with the polarization state of decaying tau lepton [2225](picture 1). For instance, in the case of mostly right-handed tau decay, due to the angular momentum conservation law, most of the tau's momentum is transferred to the pi-meson. On the contrary, in the case of mostly left-handed tau, the significant part of its momentum is transferred to the neutrino. Thereby, the energy of single pi-meson is a convenient characteristic of the polarization state of its parent tau.

One of the closest opportunities for testing this approach is the future NICA SPD experiment [26]. Since at the NICA collider a pair of tau is produced near the reaction threshold, the leptons can be at rest or possess a significant momentum, which affects the tau decay products and makes this process very sensitive to the polarization states and momenta of the initial hadrons. Hence, while conducting the research we will primarily consider the NICA conditions.

In the next section, we will demonstrate the feasibility of this approach by conducting a numerical simulation.

\section{Numerical simulation}

\subsection{Simulation details}

Numerical simulations at a centre of mass energy $\sqrt{s}=26 \mathrm{GeV}$, which corresponds to the energy of the NICA collider, have been carried out. We have performed computations of the tau production in proton-proton collisions with different polarization of initial partons. The diagram for the simulated process is shown in figure 2 (right).

Events were generated in Madgraph 5 package [27]. For the simulation of the polarized processes, we have used polarized PDF set provided by NNPDF collaboration [28]. Generated events were transferred to Pythia 8 package [29] for parton showering and hadronization. Finally, events were treated by Delphes package [30] for detector simulation and reconstruction.

\subsection{Simulation results}

The energy spectra of pions produced in the unpolarized case can be seen in picture 2 (right). Both pi-plus and pi-minus mesons manifest a similar behaviour. 

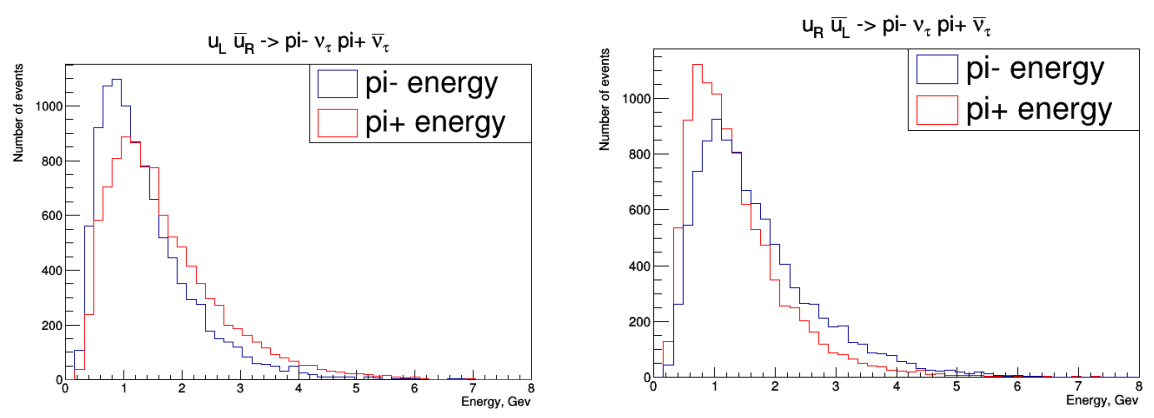

Figure 3. Energy spectra of pi-mesons produced in processes with different polarization of the initial partons: left picture corresponds to the case of left-handed quark and right-handed anti-quark, while right one corresponds to the case of right-handed quark and left-handed anti-quark

The picture becomes interesting in the case of polarized initial states. The figure 3 shows the energy spectra of pions produced in the processes with different polarization of initial partons. The first figure corresponds to the case of left-handed quark interacting with righthanded anti-quark while the second one corresponds to the case of right-handed quark interacting with left-handed anti-quark. As we can see, the energy spectra of the pions are correlating with the polarization states of quarks in the way described in the previous section. Moreover, the clear asymmetry is seen between the energy spectra of pi-plus and pi-minus mesons, which can also be utilised as a characteristic of the polarization state of the initial quarks.

Following this approach one can determine the polarization of interacting partons by measuring energies of pi-mesons produced in corresponding decays of tau-leptons. Considering the fact that pions are rather easy detectable [31], the proposed approach is potentially very convenient and powerful tool for studying PDFs via tau production in polarised Drell-Yan process.

\section{Conclusion}

We have proposed a new approach for studying parton distribution functions via tau production in polarized Drell-Yan process. The new method utilises the energy of pi-mesons produced in certain decay channels of tau-lepton as an easily measurable and convenient characteristic of the tau polarization state.

At the same time, the first steps towards developing a new potentially powerful tool for studying the internal structure of hadrons were made. The created model have shown the feasibility of such an approach and have established the basis for further development.

\section{References}

[1] S. E. Kuhn, J.-P. Chen, E. Leader, Prog.Part.Nucl.Phys. 63, 1-50 (2009)

[2] A. Deur, S. J. Brodsky, G. F. de Teramond, Rep. Prog. Phys. 82(7), (2019)

[3] A.V. Belitsky, A.V. Radyushkin, Phys.Rept. 418, 1-387 (2005)

[4] K. Goeke, M.V. Polyakov, M. Vanderhaeghen, Prog.Part.Nucl.Phys. 47, 401-515 (2001)

[5] M. Diehl, Phys.Rept. 388, 41-277 (2003)

[6] U. D’Alesio, F. Murgia, Prog.Part.Nucl.Phys. 61, 394-454 (2008) 
[7] V. Barone, A. Drago, P.G. Ratcliffe, Phys.Rept. 359, 1-168 (2002)

[8] S. Alekhin, K. Melnikov, F. Petriello, Phys.Rev.D 74, 054033 (2006)

[9] A. Bacchetta, M. Diehl, K. Goeke, A. Metz, P.J. Mulders, M. Schlegel, JHEP02 2007, 093 (2007)

[10] Compass Collaboration, PoS DIS2019 267, (2019)

[11] S. Arnold, A. Metz and M. Schlegel, Phys.Rev. D 79, 034005 (2009)

[12] A. Sissakian, O. Shevchenko, A. Nagaytsev, O. Ivanov, Phys.Rev. D 72, 054027 (2005)

[13] A. Sissakian, O. Shevchenko, A. Nagaytsev, O. Ivanov, Eur.Phys.J C 59, 659-673 (2009)

[14] A. Sissakian, O. Shevchenko, A. Nagaytsev, O. Denisov, O. Ivanov, Eur.Phys.J C 46, 147-150 (2006)

[15] A. Sissakian, O. Shevchenko, O. Ivanov, JETP Lett. 86, 751-755 (2007)

[16] A. Sissakian, O. Shevchenko, A. Nagaitsev,O. Ivanov, Physics of Particles and Nuclei 41, 64-100 (2010)

[17] J.C.Collins, D. E.Soper, G. Sterman, Nucl. Phys. B 250, 199-224 (1985)

[18] S. J. Brodsky, D. S. Hwang, I. Schmidt, Nucl.Phys. B 642, 344-356 (2002)

[19] S.D. Drell, Tung-Mow Yan, Phys.Rev.Lett. 25, 316-320 (1970)

[20] G. T. Bodwin, Phys. Rev. D 31(10), 2616-2642 (1985)

[21] J. C. Collins, Nuclear Physics B 394, 169-199 (1993)

[22] E. Boos, V. Bunichev, M. Carena, C.E.M. Wagner, ECONF C050318:0213, (2005)

[23] M. M. Nojiri, Phys. Rev. D 51, 6281 (1995)

[24] E. Boos, H. U. Martyn, G. Moortgat-Pick, M. Sachwitz, A. Sherstnev and P. M. Zerwas, Eur. Phys. J. C 30, 395 (2003)

[25] E. Boos, G. Moortgat-Pick, H. U. Martyn, M. Sachwitz and A. Vologdin, arXiv:hep$\mathrm{ph} / 0211040$

[26] I.A. Savin, A.V. Efremov, D.V. Peshekhonov, A.D. Kovalenko, O.V. Teryaev, O. Yu. Shevchenko, A.P. Nagajcev, A.V. Guskov, V.V. Kukhtin, N.D. Topilin et al., EPJ Web Conf. 85, (2015) 02039

[27] J. Alwall et al, arXiv:1405.0301 [hep-ph]

[28] The NNPDF Collaboration, Nucl.Phys. B 887, 276-308 (2014)

[29] T. Sjöstrand, S. Mrenna and P. Skands,JHEP05 026 (2006), Comput. Phys. Comm. 178 (2008)

[30] J. de Favereau, C. Delaere, P. Demin, A. Giammanco, V. Lemaître, A. Mertens, M. Selvaggi, arXiv:1307.6346 [hep-ex]

[31] CMS Collaboration, JINST 13, P10005 (2018) 